\title{
Estimulación Musical Temprana: una aproximación interdisciplinar para la integración sensorial en Educación Infantil Early Music Stimulation: An Interdisciplinary Approach For Sensory Integration In Early Childhood Education
}

\author{
Laura Miranda $^{1}$, Berta Gándara ${ }^{2}$, Olaya Pérez ${ }^{3}$, Paula Raposo ${ }^{3}$ \\ mirandalaura@uniovi.es, bertagandara@gmail.com, olayapf@gmail.com,paularraposo@gmail.com \\ ${ }^{1}$ Dpto. de Historia del Arte y \\ Musicología \\ Universidad de Oviedo \\ Oviedo, España
}

\begin{abstract}
Resumen- En este paper se resume el trabajo llevado a cabo en la asignatura "Canción Infantil y Desarrollo Integral”, impartida en la Facultad de Formación del Profesorado y Educación de la Universidad de Oviedo. Con este proyecto se promueve la interacción entre Pedagogía Musical y Terapia Ocupacional, formando al alumnado en la adquisición de competencias para relacionar el desarrollo sensorio-motor del niño y el aprendizaje musical. Para la estimulación musical temprana nos hemos basado en los principios del método Suzuki, diseñado expresamente en función de las características evolutivas del niño en edad prescolar. La aplicación de la Terapia Ocupacional se ha basado en el enfoque de la Integración sensorial de Ayres, que surge en los años sesenta de la mano de la terapeuta ocupacional Ayres para investigar las posibles relaciones establecidas entre las alteraciones en el desarrollo sensorio-motor y el aprendizaje académico, derivadas de alteraciones en el procesamiento sensorial.
\end{abstract}

Palabras clave: Estimulación Musical Temprana, Integración Sensorial, Educación Infantil

\begin{abstract}
This paper summarizes the work carried out in the subject "Canción Infantil y Desarrollo Integral", taught in the Facultad de Formación del Profesorado y Educación, University of Oviedo. This project promotes the interaction between Musical Pedagogy and Occupational Therapy, training students in the acquisition of skills to relate the child's sensory-motor development and musical learning. For early musical stimulation we have deepened on the principles of the Suzuki method, expressly designed according to the evolutionary characteristics of the child in preschool age. The application of Occupational Therapy has been based on the Ayres sensory integration approach, which emerged in the 1960s from the occupational therapist Ayres in order to investigate the relationships established between alterations in sensory-motor development and academic learning, resulting from alterations in sensory processing.
\end{abstract}

Keywords: Early Music Stimulation, Sensory Integration, Early Childhood Education

\section{INTRODUCCIÓN}

Este proyecto promueve la interacción entre Pedagogía Musical y Terapia Ocupacional mediante la asignatura "Canción Infantil y Desarrollo Integral", coordinada por Laura Miranda en la Facultad de Formación del Profesorado y Educación de la Universidad de Oviedo. Se ha formado al alumnado en la adquisición de competencias para relacionar el desarrollo sensorio-motor del niño y el aprendizaje musical. El alumnado realizó prácticas externas en las aulas de Educación Infantil de los colegios Gesta y Dolores Medio de Oviedo, donde comprobó la importancia de aunar ambas disciplinas para su futuro profesional. Este proyecto ha contado con la colaboración de cuatro entidades ajenas a la Universidad de Oviedo: 1) Colegio Gesta; 2) Colegio Dolores Medio; 3) Clínica de Terapia Ocupacional Pediátrica BeaudryBellefeuille y 4) Aula Allegretto. Berta Gándara, terapeuta ocupacional, colaboró en la formación teórico-práctica del alumnado en el procesamiento sensorial, mientras que Paula Raposo y Olaya Pérez lo hicieron en el ámbito de la Estimulación Musical Temprana.

\section{CONTEXTO}

\section{A. Objetivos}

Este proyecto incluye los objetivos generales que se detallan a continuación:

a) Innovación en el ámbito de la metodología docente. Este es un proyecto pionero en la Universidad de Oviedo y, en la actualidad, España no cuenta con trabajos destacados de investigación que relacionen el enfoque de la integración sensorial y la música.

b) Promocionar proyectos orientados al futuro mundo laboral de los estudiantes. Los alumnos han realizado prácticas en dos colegios públicos de Educación Infantil con el objetivo de potenciar sus habilidades ante su inminente futuro profesional.

c) Fomentar la coordinación y vinculación con entidades externas a la universidad. Este proyecto busca establecer una estrecha relación entre la docencia desarrollada en las aulas universitarias y la práctica profesional en centros especializados. Proponemos una colaboración entre la Universidad de Oviedo y cuatro entidades externas: los colegios públicos Gesta y Dolores Medio, el Centro de Terapia Ocupacional Pediátrica Beaudry-Bellefeuille y el centro de pedagogías activas de educación musical Aula Allegretto. 
d) Incorporar las TICs en la oferta formativa. Este proyecto ha instruido a los alumnos en el uso del programa informático Flash para la elaboración de "musicomovigramas”. El objetivo es aportar a los estudiantes una nueva herramienta psicopedagógica que relacione sistemas sensoriales con planificación y ejecución motora.

e) Incrementar de las prácticas que realizan los estudiantes, tanto las relacionadas con su carrera como en proyectos de cooperación sobre el terreno para reforzar su dimensión solidaria. En la actualidad, los colegios apuestan por la inclusión educativa en el aula, incluyendo niños con diagnósticos clínicos concretos como, por ejemplo, el trastorno del espectro autista (TEA). Desde esta perspectiva, es importante aportar a los futuros profesionales de la educación una visión más global de los niños y de sus posibles problemas en el aula, en este caso a través de la música, tan importante en Educación Infantil.

\section{B. Contexto}

El proyecto se desarrolló a lo largo del primer semestre del curso 2016-17, coincidiendo con la docencia de "Canción Infantil y Desarrollo Integral”. La asignatura, de carácter optativo, pretende formar en diversos aspectos de la música aplicados al canto a un alumnado que, en general, no tiene conocimientos musicales. Además, en el Grado en Maestro en Educación Infantil que oferta la Universidad de Oviedo no existen asignaturas específicas de Estimulación Musical Temprana. Por ende, la música no es una asignatura obligatoria en las aulas de Educación Infantil, por lo que su inclusión en el aula por parte de profesionales sin formación en el área peca de falta de rigor. La música se convierte en un "acompañante" de los niños sin una finalidad educativa clara ${ }^{1}$.

Los alumnos del Grado en Maestro en Educación Infantil presentan asimismo una carencia de conocimientos sobre Terapia Ocupacional que se ve reflejada en su posterior actividad docente, puesto que en las aulas de los colegios aumentan día a día los alumnos con TEA, DIS y otras enfermedades diagnosticadas de carácter neurológico. La música se presenta como un elemento integrador de primer orden para ayudar tanto a maestros como a alumnos en el aula, pero el docente precisa de conocimientos previos y, sobre todo, de aplicaciones prácticas.

Las clases teóricas del curso se impartieron en las aulas de la Universidad de Oviedo, incluyendo el Aula de Música de la Facultad de Formación del Profesorado y Educación. Las seis alumnas (todas mujeres) realizaron prácticas formativas en el colegio Gesta, donde desarrollaron cinco clases prácticas con niños de cuatro y cinco años, de 45 minutos cada una, con una media de 20 alumnos por aula. Un alumno diagnosticado con TEA (Síndrome Asperger) contaba con una profesora de apoyo. En el colegio Dolores Medio las alumnas asistieron a un concierto musical en el que ayudaron y también fueron espectadoras, siendo así conscientes de la importancia de la música en la cohesión de grupos de edad diversa y el interés que despierta la música en los más pequeños.

\section{Público objetivo}

\footnotetext{
${ }^{1}$ Sobre la importancia de la música en Educación Infantil véanse, entre otras, Cabrelles Sagredo, sin fecha; Díaz, 2004; Lacárcel Moreno, 1991; McMahon, 1985.
}

Alumnos del Grado de Magisterio en Educación Infantil de la Universidad de Oviedo, concretamente de la asignatura “Canción Infantil y Desarrollo Integral”.

\section{DESCRIPCIÓN}

\section{A. Tecnología}

Para este proyecto se han precisado elementos básicos de trabajo: un ordenador, un reproductor y una pantalla de proyección. Las profesoras de música han trabajado con sus propios instrumentos, además de los instrumentos presentes en el Aula de Música de la Facultad del Profesorado y Educación de la Universidad de Oviedo. Se ha promovido el empleo de las TICs a partir del programa Flash.

\section{B. Metodología}

Para la Estimulación Musical Temprana nos hemos basado en los principios del método Suzuki, diseñado expresamente en función de las características evolutivas del niño en edad prescolar (Suzuki, 1969). Suzuki diseñó su sistema basándose en los principios del aprendizaje de la lengua materna: comienzo temprano, apoyo e implicación familiar, creación de un ambiente musical, interacción musical con otros niños y planteamiento lúdico. Además, Olaya Pérez y Paula Raposo han ideado ejercicios y juegos musicales para este trabajo que se detallan en el apartado 3.D.

Tanto los aspectos teóricos como las sesiones prácticas se enmarcaron dentro del proyecto Cancionarte, que comprende las siguientes acciones:

Selección de canciones y elaboración de material propio, dirigido a niños de 3-6 años, para su uso práctico en la clase de educación musical e infantil.

- $\quad$ Realización de observaciones y prácticas en sesiones de Estimulación Musical Temprana de Aula Allegretto.

- Realización de prácticas en sesiones de Educación Infantil en el Colegio Público Gesta de Oviedo.

- Diseño de un concierto para público infantil basado en el repertorio de canciones seleccionado que integre diversas formas de expresión artística.

- Elaboración individual, por cada uno de los participantes, de diarios que recojan las impresiones personales, las expectativas, los momentos de conflicto y la valoración de la propia experiencia y del proceso de elaboración e implementación del proyecto.

Según los principios del trabajo por proyectos, la metodología empleada se basará en el enfoque globalizador, el aprendizaje interpersonal activo y la evaluación procesual a través de la práctica reflexiva.

La aplicación de la Terapia Ocupacional se ha basado en el enfoque de la Integración sensorial de Ayres, para investigar las posibles relaciones establecidas entre las alteraciones en el desarrollo sensorio-motor y el aprendizaje académico, derivadas de alteraciones en el procesamiento sensorial. La teoría de la Integración Sensorial, definida como "el proceso neurológico que organiza sensaciones procedentes del propio cuerpo y del medio ambiente, haciendo posible el uso efectivo del cuerpo en el entorno” (Jirikowic, Olson y Kartin, 2008), fue desarrollada en los años 60 por la doctora Ayres (Ayres, 1963), quien investigó la relación entre los problemas de 
interpretación sensorial y las dificultades en el desarrollo sensorio-motor y aprendizaje académico (Fisher y Murray, 1991; Bundy y Murray, 2002).

Las dificultades derivadas de una Disfunción de la Integración Sensorial (DIS) han sido relacionadas con numerosos factores de riesgo y patologías asociadas. Dentro de los factores de riesgo destacan el estrés prenatal materno (Schneider, Moore, Gajewski, Larson y Roberts, 2008; MayBenson, Koomar y Teasdale, 2009), la exposición de la madre a sustancias tóxicas (Boutros, Uretsky, Berntson y Bornstein, 1994; Fjeldsted y Hanlon-Dearman, 2009), los contaminantes ambientales (Bellinger, Leviton, Needleman, Waternaux y Rabinowitz, 1986) así como la prematuridad, las complicaciones obstétricas (Case-Smith, Butcher y Reed, 1998; Goldsmith , Van Hulle, Arneson y Schreiber, 2006; Vázquez y Bellefeuille, 2014) o el factor genético (Fjeldsted y Hanlon-Dearman, 2009). Dentro de las patologías asociadas destacan dentro de la población infantil el trastorno del espectro del autismo (TEA) (Goldsmith, Van Hulle, Arneson y Schreiber, 2006; Franklin, Deitz, Jirikowic y Astley, 2008), el trastorno por déficit de atención y comportamiento perturbador (Ghanizadeh, 2009; Holstein, Vollenweider, Geyer, Csomor, Belser y Eich, 2013) y el trastorno de eliminación como la encopresis y/o enuresis (Beaudry y Ramos, 2011; Beaudry, Schaaf y Ramos, 2013). La prevalencia de niños con DIS en países como Estados Unidos oscila entre el 5 y el 17\% (Ahn, Miller, Milberger y McIntosh, 2004; Ben-Sasson, Carter y Briggs-Gowan, 2009) y, aunque en España aún no se conocen datos de prevalencia, se estima que podrían ser similares. La prevalencia de DIS en niños con diagnósticos clínico concreto, como el TEA (Goldsmith, Van Hulle, Arneson y Schreiber, 2006; Franklin, Deitz, Jirikowic y Astley, 2008), estos datos aumentan.

\section{Técnicas}

Las clases teóricas sirvieron de apoyo a las alumnas para contar con herramientas que llevar al aula. Se les facilitó información y materiales elaborados por las autoras para que, en base a ello y a las características de las clases de infantil, las alumnas elaborasen sesiones de 45 minutos. Se trabajaron los siguientes parámetros musicales: ritmo e intensidad, a la vez que se tuvieron en cuenta las necesidades específicas de los niños según la teoría de Integración Sensorial de Ayres.

Cada sesión tiene un número diferente de actividades. Las alumnas idearon sesiones del mismo número de actividades, pero al llevarlas a la práctica, la casuística de cada clase marcó el ritmo de las actividades. Tuvieron que enfrentarse a situaciones no planificadas que también marcaron el tempo de las clases. Hemos preferido mantener las clases tal y como fueron desarrolladas in situ por las alumnas, mostrando sus capacidades organizativa y creativa en el aula de infantil, así como su capacidad a la hora de solucionar problemas concretos. Además, el hecho de no tener conocimientos musicales previos supuso un reto para ellas, que superaron con creces a la vista de la planificación de estas sesiones.

\section{Actividades}

\section{Sesión 1. 3 de noviembre de 2016}

Todas las actividades de esta sesión han sido diseñadas para trabajar el ritmo.
Actividad 1: “La lluvia”. Los niños tienen que imitar el sonido de lluvia utilizando claves, cajas chinas o las propias manos al ritmo de "El invierno" en Las cuatro estaciones (Antonio Vivaldi).

Actividad 2: "Soy un enano, soy un gigante" (Castro Ares 2017). Los niños hacen las veces de gigantes mientras suena la canción y siguen el ritmo con piernas y pies. Cuando suena el gigante, marcan el ritmo con más fuerza; cuando suena el enano, con menos.

Actividad 3: "El caballo de bronce”. Esta actividad está basada en la ópera cómica Le cheval de bronze, del compositor francés Danie Auber. El fragmento utilizado está dividido en dos partes: una con música y otra con silencio. Los niños tienen que marcar con las palmas en los muslos el ritmo mientas suena la música. Durante los momentos en silencio deben permanecer quietos.

Actividad 4: "Lento, muy lento" (Nathán, 2013). Los niños siguen el ritmo de la canción mientras deambulan por la clase, alternando partes más lentas y partes más rápidas.

Actividad 5: “Mariposa, casa, pan” (La Teacher María, 2012). Ejercicio rítmico con el que los niños practican las figuras blanca, negra y corchea, asociadas a las palabras “mariposa”, "casa y "pan”.

Actividad 6: “Aplausos” (Familia Telerín, 2016). Los niños, al igual que en la canción anterior, tienen que aplaudir al ritmo de la canción.

\section{Sesión 2. 10 de noviembre de 2016}

Todas las actividades de esta sesión han sido diseñadas para trabajar la intensidad.

Actividad 1: "El diafragma”. Los niños aprenden qué es el diafragma y descubren cómo funciona al respirar. Las alumnas comparan la respiración abdominal con hinchar un globo y los niños "prueban” a hinchar su barriga. Después emiten sonidos de diferentes intensidades mientras juegan con su diafragma.

Actividad 2: "El pandero". Los niños descubren que con el pandero pueden hacer sonidos fuertes y débiles. Se les reparte tiras de dos colores: rojo, que simboliza sonidos fuertes, y verde, para los sonidos suaves. La profesora toca con el pandero sonidos de diferentes intensidades y los niños tienes que distinguirlos.

Actividad 3: "El musicograma". Las alumnas idearon un musicograma y en la pantalla digital aparecen tres dibujos: unos zapatos, un coche y unas manos, en dos tamaños, grandes y pequeños. Los niños tienen que asociar la intensidad sonora según el tamaño del dibujo.

\section{Sesión 3. 17 de noviembre de 2016}

Todas las actividades de esta sesión han sido diseñadas para trabajar la intensidad.

Actividad 1: “La canción del acuario” (Lee, 2012). Los niños tienen que "nadar" por la clase tras auto-asignarse un animal marino mientras escuchan "Acuario" de El carnaval de los animales (Camille Saint-Saëns). Cuando la música sube de volumen tienen que agacharse, asumiendo que les acecha un tiburón. El resto de la canción permanecen nadando por la clase ad libitum. 
Actividad 2: “El pandero 2”. Los niños, que ya están familiarizados con el pandero, tienen que dar un paso atrás cuando la profesora marca un sonido suave; del mismo modo, un paso adelante cuando suena fuerte.

Actividad 3: “La alegría en el mundo". Esta actividad se trabaja con el clásico de navidad "Joy to the World" (atribuido a Haendel). Cuando la canción aumenta en intensidad, los niños tienen que dar palmadas fuertes; cuando disminuye en intensidad, palmadas suaves.

Actividad 4: “Aplausos”. Los niños cierran la sesión al ritmo de esta canción que ya han interiorizado.

\section{Sesión 4. 10 de noviembre de 2016}

Todas las actividades de esta sesión han sido diseñadas para trabajar la intensidad.

Actividad 1: “El diafragma 2". De nuevo, se trabaja con los niños la respiración diafragmática, pero en este caso las alumnas utilizaron tarjetones con los siguientes dibujos: inspirar por la nariz, hinchar la barriga, expulsar aire por la boca. De nuevo, se practica la emisión de sonidos fuertes y débiles mientras los niños comprueban la importancia capital del diafragma.

Actividad 2: “El diafragma 3”. Después de practicar la emisión de sonidos, los niños se dividen en dos bloques: unos emiten sonidos de diferentes intensidades mientras otros levantan pañuelos rojos o verdes, según la intensidad de cada sonido. En esta actividad se intercambian los papeles y se contabilizan las veces que los alumnos aciertan asociando colores a intensidades de sonidos emitidos.

Actividad 3: “Musicograma 2”. Las alumnas idearon otro musicograma para utilizar en pantalla digital con otros dibujos: unas manos, unos chasquidos y unos pies, en dos tamaños, grandes y pequeños. Los niños tienen que asociar la intensidad sonora según el tamaño del dibujo.

Actividad 4: “La canción del acuario 2". De nuevo, los niños "nadan” por la clase tras auto-asignarse un animal marino mientras escuchan "Acuario" de El carnaval de los animales (Camille Saint-Saëns). En este caso, utilizan los lazos de colores rojo y verde. Cuando suena la música principal (más fuerte) tienen que mover el lazo rojo, mientras que cuando suena la música que ellos asocian con burbujas, mueven el lazo verde.

\section{Sesión 5.17 de noviembre de 2016}

Todas las actividades de esta sesión han sido diseñadas para trabajar el ritmo, la intensidad y un nuevo elemento, el silencio.

Actividad 1: "En la sala del rey de la montaña” (Askathor, 2010). Los niños representan "In the Hall of the Mountain King” (Peer Gynt, Edvard Grieg), caminando más lentamente al principio y ganando en velocidad según avanza la obra. Del mismo modo, imaginan la pieza como una obra programática $\mathrm{y}$, por último, tras visionar el ejemplo audiovisual, comparan y contrastan sus propias versiones del asunto.

Actividad 2: “Sonidos del entorno”. Los niños tienen que imitar tres sonidos del entorno con diferentes intensidades: el claxon de un coche, una mesa contra el suelo y nuestros pies al golpear el suelo. Los niños tienen que crear después una música en grupo utilizando solo estos tres sonidos.
Actividad 3: "La primavera”. Se utiliza “La primavera” de Las cuatro estaciones (Vivaldi) para trabajar la intensidad a través de la psicomotricidad. Los niños se desplazan agachados por la clase cuando la música suena suave y muy estirados cuando suena fuerte. Cuando van agachados les decimos que son hormigas $y$, cuando van estirados, jirafas.

Actividad 4. "Pan con mantequilla" (Bellaterra Música Editorial, 2014). La canción de W.A. Mozart "Pan con mantequilla" es la excusa para imitar el acto de untar mantequilla en el pan del desayuno al ritmo de la música, mientras los niños siguen el ritmo del resto de la canción por toda la clase.

\section{Resultados}

Las actividades realizadas por las alumnas durante este proyecto, así como sus dudas, intereses y avances, han sido evaluados de manera continua. La forma de evaluar el impacto ha sido mediante la observación directa y un cuestionario en asamblea que se desarrollaba después de cada sesión en el colegio:

\section{¿Ha sido útil el abordaje metodológico?}

¿Habéis detectado patologías descritas en las clases teóricas?

¿Las habéis trabajado de manera consciente a través de la música?

¿Habéis echado de menos aspectos metodológicos en las clases teóricas para su aplicación en las sesiones?

¿Habéis detectado niños con necesidades educativas especiales?

Según declaraciones de las alumnas, este proyecto ha tenido un gran impacto, teniendo en cuenta que, hasta la fecha, la música en Educación Infantil es una rama del conocimiento muy poco explorada e infrautilizada en el aula. Las alumnas se han acercado a la asignatura con una timidez propia del desconocimiento. A lo largo de las sesiones han desarrollado un creciente interés hacia la materia y sus posibilidades, culminando en la configuración de sesiones para su aplicación en el aula. Las primeras sesiones se desarrollaron con miedo y con resultados “decepcionantes” (según el criterio de las alumnas). Sin embargo, la confianza en sus habilidades y su capacidad para detectar "problemas" en el aula fue acrecentándose sesión a sesión.

Al terminar este proyecto, todas las alumnas han manifestado su interés por continuar utilizando la música en su aula de Educación Infantil, tanto como área de trabajo interdisciplinar como recurso básico para tratar patologías como TEA y DIS.

\section{CONCLUSIONES}

La música y, en concreto, la canción es un recurso polivalente que puede utilizarse en la etapa de Educación Infantil para la Estimulación Musical Temprana, el aprendizaje de otros idiomas o, en el caso que nos ocupa, la integración de niños con patologías tales como TEA o DIS. En muchos casos no se utiliza más que como elemento complementario sin función práctica aparente debido a la falta de formación del profesorado. 
Con este proyecto se ha demostrado que alumnas del Grado en Maestro en Educación Infantil sin formación musical, pero con interés por estas áreas de estudio, han aprendido conocimientos sencillos que, no obstante, les han ayudado a incorporar la formación musical en Educación Infantil y han sido capaces de llevarla al aula. Con una guía básica sobre patologías como TEA o DIS las alumnas han adquirido habilidades para trabajar con alumnos que presentan estas patologías e, incluso, detectar algunos de sus síntomas en otros niños, promoviendo así una actitud alerta ante posibles casos sin diagnosticar. Las posibilidades de extrapolar estas enseñanzas a otros lugares de aprendizaje, no exclusivamente al ámbito escolar, confieren un carácter maleable a este proyecto, extensible a centros privados y actividades extraescolares, por citar solo algunos ejemplos.

Familiarizar a los alumnos (en general, alumnas) del Grado en Maestro en Educación Infantil con la Estimulación Musical Temprana y la Terapia Ocupacional es un primer paso para concienciar a los futuros maestros de su importancia capital en el desarrollo de las habilidades del niño y de sus posibilidades en su inminente futuro profesional y, sobre todo, emocional.

\section{AGRADECIMIENTOS}

Las autoras agradecen a las entidades participantes su colaboración desinteresada: Colegio Gesta I, Colegio Dolores Medio, Centro de Terapia Ocupacional Pediátrica Isabelle Beaudry-Bellefeuille y Aula Allegretto.

\section{REFERENCIAS}

Ahn, RR., Miller, LJ., Milberger, S., McIntosh, DN. (2004). Prevalence of parents' perceptions of sensory processing disorders among kindergarten children. Am J Occup Ther, 58(3), 287-293.

Askathor (2010, 10, 30). IN THE HALL OF THE MOUNTAIN KING - Grieg.avi. Recuperado de https://www.youtube.com/watch?v=r_Dk4oWGJQ

Ayres, AJ. (1963). The development of perceptual-motor ability: A theoretical basis for treatment of dysfunction. Am J Occup Ther, 17, 221-225.

Beaudry, IB., Ramos, EP. (2011). Tratamiento combinado de la retención voluntaria de heces mediante fármacos y terapia ocupacional. Bol Pediatr., 51(217), 169-176.

Beaudry, IB., Schaaf, RC., Ramos, EP. (2013). Brief Report: Occupational therapy based on Ayres Sensory Integration in the treatment of retentive fecal incontinence in a 3-year-old boy. Am J Occup Ther., 67(5), 601-606.

Bellaterra Música Editorial (2014, 10, 3). W.A.Mozart: "Pan con Mantequilla”. Recuperado de https://www.youtube.com/watch?v=uEh-vJoPhc0

Bellinger, D., Leviton, ANHL., Needleman, HL., Waternaux, C., Rabinowitz, M. (1986). Low-level lead exposure and infant development in the first year. Neurobehav. Toxicol. Teratol., 8(2), 151-161.

Ben-Sasson, A., Carter, S., Briggs-Gowan, MJ. (2009). Sensory Over-Responsivity in Elementary School: Prevalence and Social-Emotional Correlates. Abnorm Child Psychol., 37(5), 705-716.
Boutros, NN., Uretsky, N., Berntson, G., Bornstein, R. (1994). Effects of cocaine on sensory inhibition in rats: preliminary data. Biol Psychiatry, 36(4), 242-248.

Bröring, T., Rommelse, N., Sergeant, J., Scherder, E. (2008). Sex differences in tactile defensiveness in children with ADHD and their siblings. Dev Med Child Neurol., 50(2), 129-133.

Bundy, AC., Murray, EA. (2002). Sensory Integration: A. Jean Ayres' Theory Revisited. En AC. Bundy, SJ. Lane y EA. Murray (eds.), Sensory Integration Theory and Practice (2 ed.) (pp. 3-33) Filadelfia: F.A. Davis Company.

Cabrelles Sagredo, $\mathrm{M}^{\mathrm{a}} \mathrm{S}$. (sin fecha). El desarrollo evolutivo infantil y el juego en la educación musical. Recuperado de

http://www.docenotas.com/pdf/desarrollo_evolutivo_inf antil.pdf

Carr, JL., Agnihotri, S., Keightley, M. (2010). Sensory processing and adaptive behavior deficits of children across the fetal alcohol spectrum disorder continuum. Alcohol Clin Exp Res., 34(6), 1022-1032.

Case-Smith, J., Butcher, L., Reed, D. (1998). Parents’ report of sensory responsiveness and temperament in preterm infants. Am J Occup Ther., 52(7), 547-555.

Castro Ares, G. (2017, 6, 9). Un enano y un gigante Duendecito rojo. Recuperado de https://www.youtube.com/watch?v=0HOJi7kYENM

Díaz, M. (2004). La educación musical en la etapa 0-6 años. LÉEME 14, 1-6. Recuperado de www.musica.rediris.es/leeme/revista/diaz04.pdf

Familia Telerín (2016, 2, 1). Familia Telerín. Si Tú Tienes Muchas Ganas de Aplaudir. Canciones Infantiles para Niños. Recuperado de https://www.youtube.com/watch?v=2JbM6KJxqZg

Fisher, AG., Murray, EA. (1991). Introduction to sensory integration theory. En AG. Fisher, EA. Murray y AC Bundy, Sensory integration theory and practice (pp. 333), Filadelfia: F. A. Davis.

Fjeldsted, B., Hanlon-Dearman, A. (2009). Sensory processing and sleep challenges in children with fetal alcohol spectrum disorder. OTNow, 11(5), 26-28.

Franklin, L., Deitz, J., Jirikowic, T., Astley, S. (2008). Children with fetal alcohol spectrum disorders: Problem behaviors and sensory processing. Am J Occup Ther, 62(3), 265-273.

Ghanizadeh, A. (2009). Psychiatric comorbidity differences in clinic-referred children and adolescents with ADHD according to the subtypes and gender. J Child Neurol., 24(6), 679-684.

Goldsmith, HH., Van Hulle, CA., Arneson, CL., Schreiber, JE. (2006). A Population-Based Twin Study of Parentally Reported Tactile and Auditory Defensiveness in Young Children. J Abnorm Child Psych., 34(3), 393-407.

Gouze, KR., Hopkins, J., LeBailly, SA., Lavigne, JV. (2009). Re-examining the epidemiology of sensory regulation dysfunction and comorbid psychopathology. J Abnorm Child Psychol., 37(8), 1077-1087. 
Holstein, DH., Vollenweider, FX., Geyer, MA-, Csomor, PA., Belser, N., Eich, D. (2013). Sensory and sensorimotor gating in adult attention-deficit/hyperactivity disorder (ADHD). Psychiatry Res., 205(1), 117-126.

Jirikowic, T., Olson, CT., Kartin, D. (2008). Sensory Processing, School Performance, and Adaptive Behavior of Young School-Age Children with Fetal Alcohol Spectrum Disorders. Phys Occup Ther Pedi., 28(2), 117136.

Lacárcel Moreno, J. (1991). La psicología de la música en la educación infantil: el desarrollo musical de los cero a seis años. Revista interuniversitaria de formación del profesorado, 11, 95-110.

Lee, L. (2012, 7, 23). Camille Saint-Saens' Carnival of the Animals -Acuario. Recuperado de https://www.youtube.com/watch?v=JZVY1ZdcBqU

May-Benson, TA., Koomar, JA., Teasdale, A. (2009). Incidence of pre-, peri-, and post-natal birth and developmental problems of children with sensory processing disorder and children with autism spectrum disorder. Front Integr Neurosci., 3(31), 1-12.
McMahon, O. (1985). Young children's perceptions of the dimensions of sound. Bulletin of the Council for Research in Music Education, 85, 131-139.

Nathán, C. (2013, 3, 19). “Lento muy lento” flashmob versión. Recuperado https://www.youtube.com/watch?v=RNeO4MntTaA

Parush, S., Sohmer, H., Steinberg, A., Kaitz, M. (1997). Somatosensory functioning in children with attention deficit hyperactivity disorder. Dev Med Child Neurol., 39 (7), 464-468.

Schneider, ML., Moore, CF., Gajewski, LL., Larson, JA., Roberts, AD. (2008). Converse AK et al. Sensory processing disorder in a primate model: evidence from a longitudinal study of prenatal alcohol and prenatal stress effects. Child Dev., 79(1), 100-113.

Suzuki, S. (1969). Ability Development from Age Zero. New Jersey: Summy Birchard Inc.

Teacher María, L. (2012, 11, 5). Ejercicio musical para niños. Recuperado https://www.youtube.com/watch?v=KLSf36iPqu8

Vázquez, MC., Bellefeuille, IB. (2014) Mi-ma-me. Crecer en familia. Oviedo: Ediciones Nobel. 